\title{
Trauma und Mehrsprachigkeit in Gesellschaft und Literatur
}

\author{
Daniel WutTI \\ University College of Teacher Education Carinthia (Austria)
}

\begin{abstract}
Language is neither a neutral nor an objective medium. The context of traumatisation immediately makes clear that languages are strongly connected to people's emotional and psychological experiences. Multilingualism can help to regulate the emotional distance to stressful and traumatic experiences. At the same time, linguistic regimes, for example in the context of prestigious national languages and minority languages without prestige, have a direct impact on the psyche and self-esteem of individuals and groups. This article illustrates language
\end{abstract}

ideologies in Carinthia/Koroška, places theories from psychotraumatology in the context of contemporary multilingual literature and (un)lived social multilingualism and concludes with perspectives on flight, asylum, and migration in the context of necessary sensitivity to multilingualism.

Keywords: trauma, multilingualism, Kärnten/Koroška, emotion, minority, language ideologies

(c) by the author; daniel.wutti@ph-kaernten.ac.at

Colloquium: New Philologies, Volume 5, Issue 2 (2020)

doi: 10.23963/cnp.2020.5.2.9

Stable URL: https://colloquium.aau.at/index.php/Colloquium/article/view/140

This work is licensed under a Creative Commons Attribution 4.0 International License (CC BY 4.0). 


\section{Sprachideologien, praktisch veranschaulicht}

\subsection{Informelle und institutionalisierte Sprachideologien}

„Warum sagen wir ,Mäntschester', ,Tschikago' oder ,Marsei', wenn wir Manchester, Chicago oder Marseille meinen? Und warum sagen wir Surdum, Sahin oder Zaimoglu, obwohl die Herren oder Damen ,Schurdum', Schahin' und ,Zaimolu' heißen?", lautete die plakativ-provokative Eingangsfrage der Einladung zu einem Vortrag der Sprachwissenschafterin Katharina Brizić in Bludenz im September 2009. Die im Einladungstext unmittelbar darauffolgende Antwort lautete:

Weil wir EngländerInnen, AmerikanerInnen sowie Französinnen und Franzosen höher schätzen als Türkinnen und Türken, und weil folglich eine fehlerhafte Aussprache des Englischen oder Französischen als Ausweis von Ungebildetheit gilt, die Unfähigkeit, Türkisch richtig zu lesen, hingegen als ganz normal angesehen wird. Den Sprachen werden offenbar unterschiedliche Werte zugeschrieben und ihre Beherrschung ist ungleich viel wert. Selbst Akzente können eine Rolle spielen, wenn es um die soziale und berufliche Bewertung von Personen geht. (Verein Aktion Mitarbeit 2009)

Dieser Text kann als eine niederschwellige Einladung an die RezipientInnen gesehen werden, sich dem Diskurs von Sprachideologien zu nähern. „Sprachideologien lassen sich in [...] Spracheinstellungen, in Sprachpraktiken oder in der raumspezifischen Reglementierung von Sprachgebrauch (Sprachregime) festmachen" (Busch 2013, 81). Damit gemeint ist auch das Prestige von Sprachen zu einer jeweiligen Zeit an jeweiligen Orten: Während im deutschsprachigen Raum gegenwärtig etwa Englisch, Französisch oder Italienisch als prestigereich gelten und in weiterer Folge auch als Fremdsprachen beliebt sind (vgl. Busch 2013, 98), ist Türkisch, wie der besprochene Einladungstext nonchalant bzw. sogukkanli thematisierte, mit deutlich weniger Prestige behaftet. Entgegen dem Inhalt des oben angeführten Texts ist jedenfalls davon auszugehen, dass nicht nur die „EngländerInnen, AmerikanerInnen“ als Personen höher geschätzt werden, sondern auch damit verbundene Repräsentationen und Imaginationen „ihrer Kultur“. ${ }^{1}$ Gemäß geltender Sprachregime werden Sprachhierarchisierungen getroffen. Wie die Sprachwissenschafterin Brigitta Busch gezeigt hat, spannen sich solche Hierarchisierungen etwa von der Sprache Deutsch - an klarer erster Stelle beispielsweise bei Statistiken zu Umgangssprachen in Österreich - über die Sprachen der in Österreich anerkannten Min-

1 Keinesfalls soll der Eindruck entstehen, der Autor dieses Artikels würde einen essentialistischen Kulturbegriff vertreten, daher die Anführungszeichen. Gewiss können auch andere Faktoren zu einer Präferierung bestimmter Sprachen beitragen, wie z.B. wirtschaftliche oder geschichtliche Gründe. 
derheiten/Volksgruppen ${ }^{2}$ und den Sprachen größerer MigrantInnengruppen bis hin zu „Indianersprachen“ oder „westafrikanischen Eingeborenensprachen“ in Statistiken (vgl. Busch 2013, 16). Sprachhierarchien stehen demnach unmittelbar mit dem Prestige der jeweiligen Sprachen in Verbindung. Doch selbst die „höheren Ränge“ in solchen Kategorisierungen, wie etwa die Sprachen der in Österreich rechtlichen Sonderstatus genießenden „Volksgruppen“, werden klar getrennt von der in Österreich herrschenden „Staatssprache Deutsch“. Im zweisprachigen österreichischen Bundesland Kärnten/Koroška, in dem seit jeher (zumindest) Deutsch und Slowenisch in verschiedensten Variationen gesprochen werden, wurde diese Trennlinie zuletzt in der um den Jahresanfang 2017 teils hoch emotional geführten Diskussion um die Definition der Landessprache(n) sichtbar.

Die Debatte entfachte sich zunächst um den möglichen Passus „Die Fürsorge des Landes und der Gemeinden gilt den deutsch- und slowenischsprachigen Landsleuten gleichermaßen“ in der neu zu bestimmenden Landesverfassung: „Nach ,Bedenken in der Bevölkerung` sprach sich die ÖVP plötzlich gegen die Erwähnung der Volksgruppe in der Verfassung aus, nachdem sie die Formulierung zuvor selbst vorgeschlagen hatte" (kaernten.orf.at 2017). Als Antwort auf diesen Schwenk des damaligen ÖVP-Obmanns und Kulturlandesrats Christian Benger meldete sich u. a. das Kärntner Kulturgremium mit einer Stellungnahme, wonach es für die Kärntner Kulturschaffenden zum gelebten Selbstverständnis zähle, dass „Deutsch und Slowenisch gleichberechtigte Kärntner Landessprachen“ seien. Wie der Sprachwissenschafter Heinz-Dieter Pohl in seiner Analyse der Diskussion feststellte, ging es thematisch zunächst gar nicht um die Kärntner Landessprache(n), sie rückte(n) aber im Verlauf der Diskussion immer mehr in den Fokus (vgl. Pohl 2017, 102). ${ }^{3}$ Neben einer Vielzahl weiterer Stellungnahmen, Beiträgen und Kommentaren für oder gegen eine Erwähnung der slowenischen Minderheit und/oder Sprache in der neuen Kärntner Landesverfassung war eine der gewichtigeren gewiss jene des Verfassungsjuristen Bernd-Christian Funk, der mit Verweis auf das österreichische Bundesverfassungsgesetz ${ }^{4}$ unterstrich, Deutsch sei die einzige Staatssprache in Österreich: „Die den sprachlichen Minderheiten eingeräumten Rechte sind als Garantien von Rechten bestimmter Bevölkerungsgruppen zu verstehen. Sie bilden keine Grundlage für die Schaffung einer konkurrierenden Staatssprache im Land oder in Teilen des Landes“ (Funk,

2 Burgenlandkroatisch, Romani, Slowakisch, Slowenisch, Tschechisch und Ungarisch (hier in alphabetischer Reihenfolge). Auch diese Gruppe ist bezogen auf die gesetzliche Verankerung der Sprachen und das Prestige keinesfalls homogen.

3 Im Sinne der kritischen Diskursanalyse wäre anhand dieses Beispiels wohl von einer starken diskursiven Verschränkung zu sprechen (vgl. Jäger 2001, 97).

4 Der Artikel 8, Absatz 1 des österreichischen Bundesverfassungsgesetzes lautet: „Die deutsche Sprache ist, unbeschadet der den sprachlichen Minderheiten bundesgesetzlich eingeräumten Rechte, die Staatssprache der Republik." (Rechtsinformationssystem des Bundes 2020a). 
zitiert nach Pohl 2017, 104). Für die Kärntner Landesverfassung wurde schließlich eine Kompromisslösung gefunden, in dem die deutsche Sprache als (einzige) Landessprache (mit dem aus der Bundesverfassung entlehnten Beisatz „unbeschadet der der Minderheit bundesgesetzlich eingeräumten Rechte“), aber auch die „slowenische Volksgruppe“ (beiläufig, als Ausdruck der „gewachsenen sprachlichen und kulturellen Vielfalt“) erwähnt wird. Der slowenischen Sprache in Kärnten/Koroška bleibt in diesem Kontext immerhin der - in der gültigen Landesverfassung zwar keine Beachtung erfahrende aber dafür im österreichischen Volksgruppengesetz festgeschriebene - Status der „Amtssprache " in vielen Südkärntner Bezirken und einzelnen Südkärntner Orten. ${ }^{5}$ Das Beispiel zeigt anschaulich, dass in Kärnten/Koroška weiterhin - um mit der Sprachwissenschafterin Brigitta Busch zu sprechen - ein vom Deutschen dominiertes regionales Sprachregime herrscht. Es sind dies gesellschaftliche Fragen der Sprachpolitik, die de Cillia als „jede öffentliche Beeinflussung des Kommunikationsradius von Sprachen“ definiert (de Cillia 2003, 14, zitiert nach Busch 2013, 96). Inkludierende Sprachpolitik, die sicherstellen würde, „dass die in einer Gesellschaft gesprochenen Sprachen im öffentlichen Bereich präsent, hörbar und sichtbar sind, damit der multilinguale Charakter der Gesellschaft für alle erfahrbar wird und sich jede Person als integraler Teil der Gesellschaft wahrnehmen kann“ (Busch 2013, 124), sind dies gewiss nicht. ${ }^{6}$ Ähnlich ist die Situation gegenwärtig auch im medialen Raum Kärntens, wie im Folgenden aufzeigt wird.

\subsection{Sprachhierarchien im medialen Raum}

,Kärnten - gemeinsam in die Zukunft' - das ist das Motto für die offiziellen Feierlichkeiten des Landes am 10. Oktober. Das friedliche Zusammenleben zweier Volksgruppen in Kärnten und das Miteinander der deutschen und slowenischen Sprache stehen auch im Mittelpunkt der Gedenkfeier, mit zweisprachigen Gedanken und musikalisch unterstützt von jungen Sängerinnen und Sängern, die die Zukunft repräsentieren sollen, unter anderem der Kinderchor des Bildungszentrums Ludmannsdorf-Bilcovs. (kaernten.orf.at 2019)

5 Die konkreten Orte in Kärnten und im Burgenland, in denen als Amtssprache auch die Minderheitensprache zugelassen ist und in denen auch zweisprachige topographische Aufschriften aufzustellen sind, sind im Volksgruppengesetz geregelt (Stichwort „Ortstafellösung“) (Rechtsinformationssystem des Bundes 2020b). Grundlage dafür ist der Artikel 7 des österreichischen Staatsvertrags von 1955, der u. a. die Amtssprachen Slowenisch und Kroatisch in „den Verwaltungs- und Gerichtsbezirken Kärntens, des Burgenlands und der Steiermark mit slowenischer, kroatischer oder gemischter Bevölkerung " regelt (Rechtsinformationssystem des Bundes 1955).

${ }^{6}$ Busch bezieht sich bei dieser Definition von inkludierender Sprachenpolitik auf die Position des Advisory Committee on the Framework Convention for the Protection of National Minorities in Bezug auf Sprachenrechte (Council of Europe). 
So lautete der Einleitungstext eines Artikels der Internetredaktion des Österreichischen Rundfunks zu Feierlichkeiten am 10. Oktober 2019 in Kärnten/Koroška. Es folgte die Überschrift „Das Verbindende steht im Mittelpunkt“. Das ,Verbindende‘ sollte sowohl bei der Feier als auch im Artikel in den Vordergrund gestellt werden. Während die Worte "gemeinsam“ und „Gemeinschaft“ im Text insgesamt fünfmal vorkommen, wird die slowenische Minderheit in Kärnten, die ja Teil der beschworenen Gemeinsamkeit sein sollte, jedoch nur einmal erwähnt, nämlich in einem Direktzitat aus der Rede des gegenwärtigen Kärntner Landeshauptmanns, Peter Kaiser: „,Deutsch und slowenischsprachige Kärntner sind die Verkörperung des europäischen Grundgedankens - in Vielfalt geeint. Zeigen wir gemeinsam und mit Selbstbewusstsein, dass Kärnten großartig ist', so Kaiser“ (vgl. kaernten.orf.at 2019). Die slowenische Sprache als möglicher Teil des „gemeinsamen Kärntens" wird ebenfalls einmal im Artikel angesprochen, im bereits erwähnten Einleitungstext. In diesem wird auch ein zweisprachiger Südkärntner Ort erwähnt Ludmannsdorf, auf Slowenisch Bilčovs - jedoch fehlt im Artikel das diakritische Zeichen „ک̌ “ bei der slowenischen Bezeichnung des Ortes. Ähnlich wie bei diesem Artikel der Kärntner Redaktion des ORF zeigt sich das mediale Bild in der größten Tageszeitung im Süden Österreichs, der „Kleinen Zeitung“, im Jahr 2020: Die Zweisprachigkeit und die ethnische Identität des neuen Bischofs der Diözese Gurk, Josef Marketz, wird thematisiert, in dem der Bischof gut sichtbar mit seinem slowenischen Rufnamen angesprochen wird: „Volksbischof Josef/Joše“ (Winkler 2020, 10) - die richtige Schreibweise wäre „Jože“. Im Artikel zum Auftakt der Feierlichkeiten des Landes Kärnten zum 100. Jubiläum der Volksabstimmung im selben Medium findet sich wenige Wochen später trotz erneut vielbeschworener "Gemeinsamkeit“ weder ein slowenisches Wort noch ein „Haček“ (Bergmann 2020, 18). Bei der Veranstaltung selbst wurde fließendes Slowenisch ausschließlich von zweisprachigen Chören gesungen, in kurzen Beiträgen von Kindern und der Jugend eingebracht oder per Video zugeschaltet. Die einzige eingeladene zweisprachige Person unter den FestrednerInnen war ein international geschätzter heimischer Diplomat, der nie in der Kärntner Volksgruppenpolitik aktiv gewesen war. 
Im Jahr 2020 beabsichtigte Kärnten/Koroška unter breit angelegter Inszenierung ${ }^{7}$ die Volksabstimmung 1920, bei der deutsch- und slowenischsprachige Kärntnerinnen und Kärntner mehrheitlich für Österreich gestimmt haben, ${ }^{8}$ feierlich zu begehen. Analysen der sprachhierarchischen Situation zeigen jedoch, dass es für ein wahrhaftiges „Miteinander" noch weiterer, mutigerer Schritte bedarf. Unter anderem wird dies am nach wie vor geringen Sprachprestige des Slowenischen in Kärnten sichtbar. Sprachpolitisch wurde eine Gelegenheit zur Aufwertung eben dieses zuletzt 2017 mit der Kärntner Landesverfassung 2017 versäumt, im Jahr 2020 wurde dieses Versäumnis im Programm Carinthija 2020 offenbar wiederholt. Für ein differenziertes Verständnis, weshalb das Slowenische in Kärnten/Koroška trotz teils Jahrhunderte alter Schutzbestimmungen ${ }^{9}$ sprachideologisch noch nicht selbstverständlich ist, sollen in weiterer Folge Schnittstellen zwischen Sprache, Mehrsprachigkeit und Trauma bemüht werden.

\footnotetext{
7 Im eigens dafür eingerichteten aufwändigen Projektprogramm „CarinthiJA 2020“ wurden 89 Projekte mit einem Budget von 2,9 Millionen Euro gefördert (ktn.gv.at 2019). Mehrere der geförderten Projekte werden von ehrenamtlichen Vereinen und Initiativen getragen und zweisprachig - Deutsch und Slowenisch - ausgeführt und beworben. Die Homepage ist auf Deutsch, Slowenisch, Italienisch und Englisch gehalten - in dieser Reihenfolge zum Auswählen. Die aktuellsten und auch zahlenmäßig meisten Informationen sind auf Deutsch verfügbar. Sämtliche Projektbeschreibungen auf der Homepage carinthija2020.ktn.gv.at sind ausschließlich in deutscher Sprache verfügbar. Auch die slowenischsprachige Variante der Homepage führt - ebenso wie die italienische oder englische - zu den Projektbeschreibungen auf Deutsch. Das Land Kärnten entwickelte fürs Programm zwei Varianten des Logotyps: je nach Belieben kann dieses von Projektbeteiligten und etwa Medien zweisprachig oder deutschsprachig verwendet werden. Auf der Startseite der Homepage auf Deutsch, Italienisch und Englisch erscheint das deutschsprachige Logo. Die zweisprachige Version ist lediglich auf der slowenischen Startseite zu finden. Die slowenischen Diakritika š, č und ž werden in den Titeln der slowenischen Variante der Homepage in einem anderen „Туро“ angezeigt, obwohl die Schriftart „Metronic“ diese Diakritika grundsätzlich beinhalten würde.

8 Die Wahlbeteiligung der „Kärntner Volksabstimmung“ lag bei 95\% der Stimmberechtigten. 59,04\% stimmten für Österreich. Noch bei der Volkszählung 1910 gaben im von der Abstimmung betroffenen Gebiet 68,6\% Slowenisch als Umgangssprache an, 31,4\% Deutsch. Die Schlussfolgerung liegt nahe, dass fast jede/r Zweite mit slowenischer Umgangssprache für Österreich gestimmt haben musste (vgl. Pohl 2017: 97).

9 Bereits 1867 wurde das Staatsgrundgesetz über allgemeine Rechte der Staatsbürger eingeführt: „Die Gleichberechtigung aller landesüblichen Sprachen in Schule, Amt und öffentlichem Leben wird vom Staate anerkannt. In den Ländern, in welchen mehrere Volksstämme wohnen, sollen die öffentlichen Unterrichtsanstalten derart eingerichtet sein, daß [sic!] ohne Anwendung eines Zwanges zur Erlernung einer zweiten Landessprache jeder dieser Volksstämme die erforderlichen Mittel zur Ausbildung in seiner Sprache erhält", hieß es demnach im Artikel 19 (Rechtsinformationssystem des Bundes 2020c). Slowenisch wurde in Kärnten/Koroška bereits zuvor an Schulen unterrichtet: Die Allgemeine Schulordnung für die deutschen Normal-, Haupt-und Trivialschulen in sämtlichen k. k. Erbländern regelte sog. Utraquistische Schulen. Der Unterricht in Südkärnten erfolgte in solchen in den ersten Jahren auf Slowenisch, um die Kinder bald zur deutschen Sprache zu führen.
} 


\section{Sprache und Trauma}

\section{1 „Gewählte“ Traumata und gewählte Einsprachigkeit}

Der türkisch-zypriotische Psychoanalytiker Vamik Volkan brachte das „gewählte Trauma" (engl. chosen trauma) in den wissenschaftlichen Diskurs. Gewählte Traumata seien demnach einer von sieben Fäden der Großgruppenidentität und würden - neben den weiteren sechs Fäden, unter denen etwa auch gewählte „glorreiche Kapitel“ aus der Vergangenheit einer Gruppe zu finden sind - die Identität von Großgruppen (etwa im Sinne von Nationen oder auch Ethnien) festigen (vgl. Volkan 2005, 39).

Volkans Auffassung von „Großgruppen“ reiht sich als konstruktivistische Auffassung neben bspw. Benedict Andersons ursprünglichen Vorschlag, Nationen und Gemeinschaften als „imagined communities “10 aufzufassen, ein. Für Volkan stehen gewählte Traumata für geistige Repräsentanzen von Ereignissen, die dazu führten, dass eine Großgruppe durch eine andere (Groß-)Gruppe schwere Verluste hinnehmen musste, sich dadurch hilflos und als Opfer fühlte und demütigende Verletzungen miteinander zu teilen hatte (Volkan 1999, 73). Von politischen Führern werden solche Ereignisse bewusst gepflegt, um die kollektive Identität der Gruppe zu stärken (Ottomeyer 2009, 66). Diese „traumatischen“"Ereignisse können laut Volkan Jahrhunderte zurückliegen und dennoch außerordentlich bedeutend fürs Etablieren oder Aufrechterhalten der „Identität“ einer Gruppe sein, womit insbesondere die Vorstellung einzelner Mitglieder der Gruppe gemeint ist, sie würden zu einem größeren Ganzen gehören, also zu einer Gruppe, die sich in ihrer Einheit und Eigenart von anderen Gruppen unterscheidet.

Im Kontext des zwei- und mehrsprachigen Bundeslands Kärnten/Koroška sind gewählte Traumata wohl Gebietsansprüche Jugoslawiens bzw. des SHS-Staats an den slowenischsprachigen Teil Südkärntens nach dem Ersten und Zweiten Weltkrieg. ${ }^{11}$ Die partielle Okkupation Südkärntens seitens jugoslawischer Streitkräfte (im Gedächtnisdiskurs Sloweniens unter dem Begriff „Kampf um die Nordgrenze“ einzureihen) führte in Kärnten zur Bildung von Freiwilligenverbänden ab Ende 1918 (Danglmaier \& Koroschitz 2020, 23-24). Diese spielen noch gegenwärtig als „Abwehrkämpfer“ im kollektiven Gedächtnis Kärntens eine gewichtige Rolle - dass unter den AbwehrkämpferInnen auch Kärntner mit slowenischer Umgangssprache und Kärntner Juden waren, umso weniger. Der Erste und der Zweite Weltkrieg wurden im deutschnational geprägten Kärntner Ge-

\footnotetext{
${ }^{10}$ So schreibt Anderson: "It is an imagined political community - and imagined as both inherently limited and sovereign.” (Anderson 2006: 6). Eigentlich sei jegliche Gemeinschaft, die persönlichen Kontakt ("face-to-face") übersteigt, demnach "imagined" (Anderson 2006, 6).

${ }^{11}$ Für eine Sichtweise auf das „kulturelle Gedächtnis“ von Mehrheit und Minderheit sowie Schnittstellen zwischen Gedächtnis, Trauma und Identität und die Hierarchie der Erinnerungen in Kärnten/Koroška siehe bspw. Wutti 2017.
} 
dächtnisdiskurs zu Daten, die vom Kärntner „Abwehrkampf“ Legitimation und Sinn bekamen und die durch den ideologischen Kitt von Heldentum und Heimattreue zusammengehalten werden. Peter Gstettner beschrieb diese bemerkenswerte Diskursverschränkung, indem er den Begriff der Kärntner „Erinnerungstroika“ prägte (Gstettner 2011, 105). Die Macht dieser diskursiven Verschränkung trug in den Nachkriegsjahrzehnten sicher auch dazu bei, eine kritische Bearbeitung von Verstrickungen und Täterschaft im Nationalsozialismus weitläufig zu umgehen. Die jährlichen Feiern zum 10. Oktober dienten dabei als besondere Plattform, die „Erinnerungstroika“ gesellschaftsfähig und relevant zu halten.

Gemäß der beschriebenen („Deutschkärntner“) Auslegung der Zeitgeschichte diente das Slowenische und dabei auch insbesondere die slowenische Sprache in allen Sprachvariationen ${ }^{12}$ in Kärnten als Marker, von dem man sich abgrenzte, um die eigene Großgruppenidentität zu stärken. Im Kontext des zwei- und mehrsprachigen Bundeslands Kärnten wurde also dessen gewachsene Zweisprachigkeit zum Marker für das „Andere“, für die Alterität, zum Ursprung der (gewählten) Traumata. Wie der Zeithistoriker Helmut Konrad treffender Weise schreibt, hätte das Volksabstimmungsergebnis durchaus das Potential, Kärnten als einen gemeinsamen Erinnerungsort zu formen (vgl. Konrad 2018, 85). Bis in die 2010er Jahre blieb dieses Potential nicht nur ungenutzt. Die Feierlichkeiten um den 10. Oktober dienten dem diametral gegenüberstehend zur Aufrechterhaltung einer Ideologie der Homogenisierung in ethnischer und sprachlicher Hinsicht. Opportun für die Kärntner und Kärntnerinnen war die selbstauferlegte, „gewählte“ Einsprachigkeit, eine Flucht aus Ambivalenzen.

\subsection{Gewähltes Trauma, „reales“ Trauma und Mehrsprachigkeit}

Gewählte Traumata sind jedenfalls von realen Traumatisierungen zu unterscheiden: „Es gibt Menschen, die das Trauma erlebt und erlitten haben, und es gibt die, für die es nur ein relevantes Ereignis ist“, schreibt Angela Kühner $(2008,275)$ in Bezugnahme auf Volkans Konzept. Manche Menschen können keinesfalls wählen, ob und inwieweit ein traumatisches Ereignis für sie relevant sei. Andere, die bspw. nicht persönlich traumatisierende Sequenzen erlebt haben, können hingegen je nach Belieben ein Ereignis als für sie mehr oder weniger relevant einstufen oder sich auch einfach ,mitgemeint ' (auch als in ihrer Großgruppenidentität angesprochen) fühlen. Kühner schlussfolgert, es gebe in diesem Sinne unterschiedliche Grade von Betroffenheit (ibid., 276). ${ }^{13}$

\footnotetext{
${ }^{12}$ Eine Ausnahme dabei stellte sicher das „Windische“ dar - weniger als Sprachvariation des Slowenischen (was es keinesfalls ist), sondern mehr als eigene ethnische, soziale Kategorie zwischen den „Deutschen“ und „(National-)Slowenen“.

${ }^{13}$ Der Autor dieses Artikels beschreibt an anderer Stelle am Beispiel der Kärntner SlowenInnen, dass gewählte Traumata durchaus auch auf reale Traumata treffen können. So stellt die nationalsozialistische
} 
Traumatisierung kann aus einer humanistischen Sichtweise als eine „extreme Spontaneitätsstörung“ beschrieben werden (Ottomeyer 2004, 351). Traumatisierung sei ein Zerbrechen der (Lebens-)Erzählung(ibid., 350), ein Zerbrechen des biographischen Narrativs als „sinnstiftendes Erzählmotiv“ eines Menschen. Traumatisierungen zersetzen Sprache, das Erlebte wird unaussprechbar. Traumatische Erfahrungen im Sinne extremer Grenzerfahrungen zeichnen sich nicht zuletzt dadurch aus, dass sie die psychische Verarbeitungsfähigkeit betroffener Menschen übersteigen. Sie setzen sich daher zumeist außerhalb der sprachlich fassbaren Erinnerung ab. Traumatisierten Menschen ist es bis zur Integration des Erlebten, bspw. im Rahmen von Psychotherapie, oft nicht möglich, die traumatischen Sequenzen in Worte zu fassen. Das Trauma setzt sich tief im körperlichen Erleben des Menschen nieder. Das nicht Aussprechbare findet psychosomatisch einen Weg, sich auszudrücken, die Symptome werden körperlich. ${ }^{14}$

Dem gegenüber sind gewählte Traumata gewiss nicht sprachentzogen. Sie werden von politischen Demagogen Kraft ideologisierter machtvoller Sprache konstruiert und instrumentalisiert. Mit ihnen kann aber auch - mit einer bewusst kritischen Distanz - gewitzelt oder gespielt werden; Sprachspiele werden möglich, was im Falle realer Traumatisierungen erst nach umfangreicher Integration des schrecklichen Erlebten unter Umständen auch als zielführender Perspektivenwechsel möglich ist. Schwarzer Humor, Ironie oder Satire spielen in diesem Sinne auch mit belastenden Thematiken und helfen unter Umständen, diese greifbarer zu gestalten. Ein jüngerer Meister kreativer Sprachspiele vor dem Hintergrund (auch) belastender Thematiken ist wohl der Autor Saša Stanišić. Er spielt in seinen Texten mit mehrsprachigem Sprachwitz, er spielt mit seiner eigenen Mehrsprachigkeit und schafft auch mehrsprachige Charaktere. Er setzt Mehrsprachigkeit und mehrsprachigen Humor durchaus auch ein, um Distanz zu belastende(re)n Thematiken zu schaffen. Stanišić selbst flüchtete 1992 mit seinen Eltern mit vierzehn Jahren vor dem Jugoslawienkrieg aus Bosnien nach Deutschland. In seinen international erfolgreichen Werken bearbeitet er über den Umweg der Fiktion auch seine eigene Biografie.

Deportation slowenischer Familien im April 1942 ein identitätsstiftendes, also Gruppenidentität konstituierendes Element dar. Zugleich leben auch im Jahr 2020 noch in manchen Familien unmittelbare ZeitzeugInnen dieser Deportationen (Wutti 2015, 39); Ihre Erfahrungen wurden vielfach transgenerational tradiert, teils mit direkten Traumasymptomen, teils als mehr oder weniger wirksame Belastungen (Wutti 2013).

${ }^{14}$ Die klinischen Symptome einer Posttraumatischen Belastungsstörung werden unterteilt in zumindest Hyperreagibilität (Übererregung), Intrusive Symptome (eindringliche Erinnerungen, „Wiedererlebend") und Konstriktive Symptome (Vermeidungsverhalten) - siehe dazu bspw. Falkai \& Wittchen 2015, 168-169. Aus einer kritischen Sichtweise ist diese Kategorisierung ein zu einer jeweiligen Zeit gültiger medizinischer Konsens, mit dem versucht wird, Krankheitsbildern bzw. Symptomen zu entsprechen, und jedenfalls insofern problematisch, weil sie Traumata nach wie vor insbesondere individuell bzw. intrapersonal begreifen, was auch bspw. das Schema ICD-11 ab 2022 nicht grundlegend ändern wird (vgl. Wutti 2020). 
„Stanisic [sic!] humorously and effectively employs his (and his protagonist's) unique perspective as a multilingual individual and refugee to draw meaningful connections between Bosnian and German traumas", fasst demnach die Sprach- und Literaturwissenschafterin Didem Uca in einer Analyse von Stanišićs Werk Wie der Soldat das Grammofon repariert aus dem Jahr 2006 zusammen (Uca 2019, 189). Ucas These mag aus literaturwissenschaftlicher Sicht sinnvoll erscheinen. In ihrer nicht weiter differenzierten Verwendung des Begriffs "Trauma“ wie auch in der undifferenzierten Gleichgewichtung „deutscher Traumata“ mit Traumata aus dem Jugoslawienkrieg trägt die Autorin allerdings leider auch stark zum zu kritisierenden „Traumaboom“ (Becker 2014, 8) bei. Statt undifferenziert von kollektiven Traumata auszugehen - was Uca wohl tut - wäre es in diesem Kontext angebrachter, von Traumadiskursen, auf die sich Stanišić subtil bezieht, oder auch einfach von Belastungen zu sprechen. ${ }^{15}$ Im Fall des Jugoslawienkriegs wäre es jedenfalls möglich, von kollektivisierten Traumata auszugehen: also von mehreren individuellen (realen) Traumata, die gesellschaftliche Wirkkraft entwickeln und so auch auf Großgruppenidentitäten wirken ${ }^{16}$, was inhaltlich bereits erneut in Richtung „gewählter Traumata" ginge.

\subsection{Das Trauma in der mehr- und anderssprachigen Literatur}

Obwohl Stanišić durch die Ereignisse vor seiner Flucht aus Jugoslawien und den Jugoslawienkrieg gewiss biographisch belastet ist - hierbei wird bewusst nicht der Begriff traumatisiert verwendet - ist anzumerken, dass er in seinen Texten wohl eher humoristische Stilmittel verwendet als etwa den Sprachwechsel, um sich von persönlich belastende(re)n Thematiken in seinen Texten zu distanzieren ${ }^{17}$. Die ebenfalls mehrsprachige und gewiss durch familiär tradierte traumatische Erfahrungen belastete Kärntner slowe-

\footnotetext{
${ }^{15}$ Etwas konkreter wird der Traumabegriff in der Analyse desselben Texts von Stanišić bei Svetlana Arnaudova verwendet. Auch sie interpretiert - wie Uca - den Verweis auf Celans Todesfuge an einer Stelle als intendierten intertextuellen Bezug zwischen dem Grauen des Jugoslawienkriegs und dem Holocaust und immerhin spricht sie dabei nicht von "german trauma“ (Arnaudova 2019, 51). Arnaudova bemüht sich mithilfe von Cathy Caruth auch mehr um eine theoretische Verortung des literaturwissenschaftlichen Traumabegriffs, der aber vor dem hier in Ansätzen beschriebenen umfangreichen theoretischen Traumadiskurs dennoch undifferenziert bleibt.

${ }^{16}$ Der Sozialpsychologe Markus Brunner schlug vor, den Begriff des „kollektiven Traumas“ generell durch drei verschiedene Aspekte zu ersetzen (Brunner 2010, 10): durch die kollektive Verarbeitung massenhafter Individualtraumata, durch kollektive Traumanarrationen oder auch Traumadiskurse (was auf das von Uda Intendierte zutreffen würde) und traumainduzierte Kollektive, wobei er sich auf Angela Kühner bezog.

${ }^{17}$ Bisher unerwähnt blieb die wohl auch belastende Migrationserfahrung mitsamt ihren Herausforderungen der „Akkulturation“ und Hybridität, die als Thema in Stanišićs Texten mindestens so prominent ist wie der Jugoslawienkrieg. Oft vermischen sich im Migrationskontext belastende oder gar traumatisierende Erfahrungen vor der Flucht mit noch später großen Belastungen nach der Migration. Bei Migration vorschnell von „Trauma“ zu sprechen ist jedenfalls ebenso unkorrekt wie die alleinige Fokussierung auf den Krieg als belastende oder auch traumatische Erfahrung.
} 
nische Autorin Maja Haderlap folgt einer anderen Strategie. Ihr Roman Engel des Vergessens, für den sie den renommierten Ingeborg-Bachmann-Preis und in der Folge eine Reihe weiterer prestigereicher Literaturpreise verliehen bekam, erschien 2011 zunächst auf Deutsch und wurde erst später von einem professionellen Übersetzer auf Slowenisch - der Mutter- bzw. Erstsprache der Autorin - übersetzt (Gračner 2016, 96). Inzwischen ist die slowenische Übersetzung des Romans in Slowenien Pflichtliteratur für die Sekundarstufe und auch Maturastoff. Auf die Frage, weshalb sie den Roman nicht auf Slowenisch verfasst habe, meint die Schriftstellerin im Interview mit der „Presse“: „Ich hätte den Roman nicht auf Slowenisch schreiben können. Mir ist das Deutsche inzwischen schon sehr zugewachsen, auch über die Arbeit. Es hält mich auf Distanz zu schmerzvollen Bereichen" (Mayer 2012).

Jemand, der trotz mit der slowenischen Sprache eng verknüpften biographischen Traumata bewusst auf Slowenisch schreibt, ist der Kärntner slowenische Autor Florjan Lipuš. Lipuš wurde 1937 geboren und wuchs wie Maja Haderlap bei Bad Eisenkappel/Železna Kapla in Südkärnten auf. Als er sechs Jahre alt war, wurde seine Mutter von als Partisanen verkleideten Männern der Gestapo überlistet und in weiterer Folge im KZ Ravensbrück ermordet. Wie der Laibacher Literaturhistoriker Boris Paternu beschreibt, liegt Lipušs literarischem Werk seit seinem ersten, 1964 erschienenen Buch Črtice mimogrede ein „Kärntner Sprachtrauma“ zugrunde, mit Ursprung in den Jahren seiner Kindheit während des Nationalsozialismus (Paternu 2003, 24). Lipuš schreibt seine Romane ausschließlich in slowenischer Sprache. Nachdem ihm Teile des Kunstsenats 2016 den Großen Österreichischen Staatspreis für Literatur, die bedeutendste literarische Auszeichnung des Landes, verweigerten, weil er nicht auf Deutsch schreibt, wurde ihm dieser Preis 2018 dennoch verliehen. 2019 folgte ein ähnlich hoch dotierter Preis der Republik Slowenien. Bei seiner diesbezüglichen Dankesrede zitierte Lipuš Prešern: „Z jezikom smo ali nismo, z jezikom bomo ali ne bomo" (Mit der Sprache sind wir oder sind wir nicht, mit der Sprache werden wir oder werden wir nicht sein). Das alleinige Schreiben in slowenischer Sprache sieht Lipuš als politischen Ausdruck und als seinen Beitrag für den Erhalt des Slowenischen in Kärnten (spz.slo.at 2013). Bei seiner Dankesrede zum Erhalt des oben erwähnten österreichischen Preises leitete er folgendermaßen ein:

Die Sprache ist mehr als ein Werkzeug, mehr als eine Begleiterscheinung des Alltags. Die Sprache ist nicht nur ein Unterscheidungsmerkmal, sie ist auch das einzige geistige Fundament, auf dem eine Identität errichtet werden kann. Wenn diese Erkenntnis für die Mehrheitsbevölkerung gilt, so gilt sie umso mehr für die Minderheit. Die Volksgruppe kann ihren Weiterbestand nur auf Kultur und Sprache aufbauen. Die Kärntner Slowenen haben beides zu verlieren. In der Zeit um die Kärntner Volksabstimmung, nach 
dem Ersten Weltkrieg, war ein Drittel der Kärntner Bevölkerung slowenischsprachig, heute ist die Sprache in den ehemals slowenischen Dörfern nur noch vereinzelt zu hören. Das ist jedoch nicht der alleinige Grund, warum jemand in Kärnten in seiner slowenischen Muttersprache schreibt. Es geht auch und es geht vor allem um sein persönliches Befinden, um die innere Notwendigkeit, um das Verwirklichen des Einzigartigen, des Eigenen, des Persönlichen. Die heutige Würdigung dessen empfinde ich auch als eine Wiedergutmachung des Unrechts an den Kärntner Slowenen. Während der NS-Zeit wurden über 200 slowenische Familien in Vernichtungslager gebracht oder wurden ausgesiedelt. Und sind die Zeichen der Zeit heute andere? (derstandard.at 2018)

Lipuš schloss seine Rede mit den Worten, „Sie haben die slowenische Sprache nicht nur legitimiert, nicht nur aufgewertet, Sie haben sie auch geadelt. Slowenisch ist Österreich zumutbar" (ibid.). In einem Interview mit der in Ljubljana erscheinenden Zeitschrift „Mladina“ breitete Maja Haderlap weiterführend aus, sie sei als Kärntner Slowenin mit dem Gefühl aufgewachsen, ständig ihre Existenz beweisen zu müssen:

Als sei dies nichts Normales, dass ich existiere, sondern dass ich jedem meine Vergangenheit rezitieren muss und jedem erklären, weshalb ich auf der Welt bin. Diese Gesellschaft hat einfach nicht akzeptiert, dass ich als Slowenin auch im deutschen Sprachraum bestehe. Erst jetzt ist sie darauf vorbereitet. Und auch in Slowenien muss ich mich ständig selbst legitimieren. Alles, was ich tue, weshalb ich auf Deutsch schreibe, weshalb dies und jenes. (Haderlap, zitiert nach Horvat 2011, o.S.)

Im Nationalsozialismus war die andere, fremde, in diesem Fall slowenische Sprache in Kärnten eine der gewichtigsten Ursachen für Diskriminierung. Ein großer Anteil Slowenisch sprechender Kärntnerinnen und Kärntner wählte daher den systematisch angebotenen Weg des geringsten Widerstands, verwendete die verbotene slowenische Sprache nicht mehr und gab die slowenische Sprache auch nach dem Zerfall des Nationalsozialismus nicht mehr an die Nachkommen weiter. Ein Wechsel von der Slowenisch-Deutschen Mehrsprachigkeit in die „Einsprachigkeit“ des Deutschen öffnete den Betroffenen Auswege aus der Diskriminierung. Doch auch nach dem Nationalsozialismus blieben der Druck und das Stigma durch die Sprache bestehen (vgl. Wutti 2013).

In den 1970er Jahren des vergangenen Jahrhunderts entwickelte der Arzt und Psychoanalytiker Hans Keilson nach umfangreichen Untersuchungen mit jüdischen Kinderüberlebenden des Nazi-Terrors die Theorie der sequenziellen Traumatisierung. Dabei wandte sich Keilson dem traumatischen Prozess zu, der in seiner Gesamtheit zu sehen 
ist, um Traumatisierungen verstehen zu können (vgl. Kestenberg 2016, 85). So wurde deutlich, dass der Verlauf nach der Traumatisierung - die dritte Sequenz - für die Gesundheitsperspektiven der Opfer bedeutsamer sein kann als das „eigentliche“ Trauma in der zweiten Sequenz (vgl. Becker 2014, 176). Auch kann bedeutend sein, ob das Opfer bereits zuvor ein belastendes oder eher ein ressourcenreiches Leben hatte (erste Sequenz). Bedeutend ist demnach auch, ob spätere Opfer einer Traumatisierung bereits zuvor als ethnische, religiöse oder sprachliche Minderheiten diskriminiert wurden - und insbesondere auch, wie der spätere Verlauf in der dritten Sequenz, beispielsweise also der Versuch der Traumatisierten, erneut in ein gesellschaftliches Alltagsleben zurückzufinden, verläuft. So nennt Ottomeyer die Psychotherapie mit Kärntner slowenischen Opfern des Nationalsozialismus etwa Therapie „unter schwierigen Bedingungen“, weil das Leid der ehemaligen NS-Opfer „länger als ein halbes Jahrhundert verleugnet, verschwiegen und bagatellisiert wurde und weil die gesellschaftliche Abwehr gegenüber ihren Erfahrungen immer noch groß ist“" (Ottomeyer 2011, 135).

\section{Resümee und Ausblick}

Die Theorie der traumatischen Sequenzen ist bedeutend, weil sie die soziale Bedingtheit von Traumata in den Vordergrund stellt. Sie versteht die Wirkung von Traumata nicht als vorschnell begrenz- und abschließbar, sondern unterstreicht, dass es sich bei Traumata um lange, komplexe Prozesse handelt, für die alle - gesellschaftlich - weiter Verantwortung tragen (Becker 2014, 184). Die genannten Beispiele aus dem zwei- und mehrsprachigen Bundesland Kärnten/Koroška unterstreichen die Bedeutung sozialer Verantwortung für die Integration von Traumata - oder auch ihre Perpetuierung. Die Theorie der traumatischen Sequenzen ist jedoch nicht nur anwendbar, um psychisches Leid der Opfer des Nationalsozialismus zu erklären - und das ihrer Nachkommen, die sich ebenso in einem traumatischen Zirkel wiederfinden können (Wutti 2014, 121) - sondern ebenso auf aus Krisen- und Kriegsgebiete geflüchtete Menschen. Auch in Asylverfahren wird leider wenig Wert auf psychotraumatologische Sensibilität gelegt. Sprache bzw. das Sprechen beruht auf gegenseitiger Anerkennung, dem gegenübergesetzt entsteht durch sprachliche Gewalt aber auch Entsubjektivierung. So ist es für Flüchtlinge beim Erlernen neuer Sprache(n) im Aufnahmeland überaus fördernd, wenn klare Verhältnisse vorherrschen, also bspw. ein fixer rechtlichen Status und anerkannter Standort (Busch 2016, 100). Jahrelang andauernde Asylverfahren erschweren dies aber erheblich. Erschwerend ist für traumatisierte Flüchtlinge auch die institutionelle Kommunikation im Rahmen ihrer Asylverfahren (Busch 2013, 166). 
Mehrsprachigkeit kann dabei helfen, die emotionale Nähe zu schwierigen (auch traumatischen) Erlebnissen zu regulieren, was, wie gezeigt wurde, etwa literarische AutorInnen (mehr oder weniger) bewusst anwenden. Sprachliche Regime, etwa im Kontext von prestigereichen Staatssprachen und Minderheitensprachen ohne Prestige, wirken sich unmittelbar auf die Psyche und das Selbstwertgefühl Einzelner aus. Abseits bedeutender Thematiken - wie ein Staat beispielsweise mit seinen anerkannten oder nur geduldeten Minderheitensprachen umgeht, ob sich ein Staat als per se ein- oder mehrsprachig begreift und ob Mehrsprachigkeit im Schulsystem als Potential gesehen wird - geht es aber nicht selten auch um existenzielle Fragen, wie bspw., ob ein diskriminierter, vielleicht auch schwer traumatisierter geflüchteter Mensch aufgrund von Unwissenheit oder auch in Ermangelung einer Sensibilität im institutionellen System von falschen DolmetscherInnen übersetzt wird. Eine zunehmende gesellschaftliche Achtsamkeit bezüglich der Themen Mehrsprachigkeit und Traumata ist angebracht.

\section{Literatur}

Anderson, B. 2006. Imagined Communities. Reflections on the Origin and Spread of Nationalism. London/New York: Verso.

Arnaudova, S. 2019. „Vergangenheitsbewältigung und Identitätskonstruktion im Roman von Saša Stanišić Wie der Soldat das Grammofon repariert." In Auswanderung und Identität. Erfahrungen von Exil, Flucht und Migration in der deutschsprachigen Literatur, herausgegeben von Ch. Baltes-Löhr, B. P. Kory und G. Sandor, 39-54. Bielefeld: Transkript.

Becker, D. 2014. Die Erfindung des Traumas. Verflochtene Geschichten. Gießen: Psychosozial.

Bergmann, A. 2020. „Kärntens Aufgabe sind der Dialog und die Kooperation. 18-19. Kleine Zeitung, 4. März 2020.

Busch, B. 2013. Mehrsprachigkeit. Wien: Facultas.

Busch, B. 2016. „Sprachliche Verletzung, verletzte Sprache: Über den Zusammenhang von traumatischem Erleben und Spracherleben." In Osnabrücker Beiträge zur Sprachtheorie (OBST) 89: $85-108$.

Brunner, M. 2010. Markus Brunner: Zum Begriff des 'kollektiven Traumas'. Oder: Wie angemessen über das Leid von Menschen sprechen in Zeiten inflationierender Opferdiskurse? Abgerufen am 2. April 2020. http://www.agpolpsy.de/wp-content/uploads/2010/06/vortrag-kollektive-trau mata-sfu.pdf

Council of Europe. o.J. „Advisory Committee on the Framework Convention for the Protection of National Minorities." Abgerufen am 02. April 2020. https://www.coe.int/en/web/ minorities/advisory-committee

Danglmaier, N. und Koroschitz, W. 2020. Nationalsozialismus in Kärnten. Opfer. Täter. Gegner. Innsbruck: Studienverlag. 
derstandard.at. 2018. „Autor Florjan Lipus: Slowenisch ist Österreich zumutbar.“ Abgerufen am 02.April 2020. https://www.derstandard.at/story/2000090533738/autor-florjan-lipusslowe nisch-ist-oesterreich-zumutbar

Falkai, P. und Wittchen, H-U. 2015. Diagnostische Kriterien DSM-5. Göttingen: Hogrefe.

Gračner, U. 2016. „Osebni, kolektivni spomin in identiteta v sodobnem slovenskem romanopisju na avstrijskem Koroškem." In Colloquium: New Philologies, 1 (1): 89-105.

Gstettner, P. 2011. Erinnern an das Vergessen, Gedächtnispolitik und Bildungspolitik. Klagenfurt/ Celovec: Kitab.

Horvat, M. 2011. „Maja Haderlap: ,Rasla sem z občutkom, da moram ves čas dokazovati svojo eksistenco؛. Pisateljica in Pesnica“" Abgerufen am 2. April 2020. https://www.mladina.si/86695/ maja-haderlap-rasla-sem-z-obcutkom-da-moram-ves-cas-dokazovati-svojo-eksistenco

Jäger, S. 2001. „Diskurs und Wissen. Theoretische und methodische Aspekte einer Kritischen Diskurs- und Dispositivanalyse." In Handbuch Sozialwissenschaftliche Diskursanalyse, Band I: Theorien und Methoden, herausgegeben von R. Keller, A. Hirseland, W. Schneider und W. Viehöver, 81-112. Opladen: Leske \& Budrich.

kaernten.orf.at. 2017. „Einigung über, Slowenenpassus““. Abgerufen am 2. April 2020. https:// kaernten.orf.at/v2/news/stories/2824970/

kaernten.orf.at. 2019. Abgerufen am 2. April. 2020. https://kaernten.orf.at/stories/3016576/

Kestenberg, M. 2016. „Diskriminierende Aspekte der deutschen Entschädigungspraxis: Eine Fortsetzung der Verfolgung." In Kinder der Opfer, Kinder der Täter. Psychoanalyse und Holocaust, herausgegeben von M. S. Bergmann, M. E. Jucovy und J. S. Kestenberg, 74-102. Frankfurt/Main: Fischer.

Konrad, H. 2018. „Identität(en) und Erinnerungskultur(en).“ In Einführung - Überblick - Reflexionen zum neuen Landesausstellungsformat, herausgegeben von Land Kärnten, 79-89.

ktn.gv.at. 2019. „CARINTHIja 2020: 89 Projekte des Jubiläumsjahres verbinden Vergangenes und Zukunft." Abgerufen am 2. April 2020. https://www.ktn.gv.at/Service/News?nid=30385

Kühner, A. 2008. Trauma und Kollektives Gedächtnis. Gießen: Psychosozial.

Lind, M. 2006. „Dolmetschunterstütztes Psychodrama mit traumatisierten Asylwerberinnen. Begegnung und Stabilisierung." In Zeitschrift für Psychodrama und Soziometrie 2/2006: 225-238.

Mayer, N. 2012. „Haderlap: ,Deutsch hält mich auf Distanz zum Schmerz““ Abgerufen am 2. April 2020. https://www.diepresse.com/743059/haderlap-deutsch-halt-mich-auf-distanz-zumschmerz.

Ottomeyer, K. 2004. „Psychodrama und Trauma.“ In Psychodrama-Therapie. Ein Handbuch, herausgegeben von J. Fürst, K. Ottomeyer und H. Pruckner, 348-362. Wien: Facultas.

Ottomeyer, K. 2009. Jörg Haider - Mythenbildung und Erbschaft. Klagenfurt/Celovec: Drava.

Ottomeyer, K. 2011. Die Behandlung der Opfer. Über unseren Umgang mit dem Trauma der Flüchtlinge und Verfolgten. Stuttgart: Klett-Cotta.

Paternu, B. 2003. „Lipušev novi preboj regionalizma.“ In Jezik in slovstvo 48/5: 19-24.

Pohl, H.-D. 2017. „Zur Diskussion um die Kärntner „Landessprache(n).“ In Kärntner Jahrbuch für Politik 2017, herausgegeben von K. Anderwald, P. Filzmaier und K. Hren, 93-115. Klagenfurt/Celovec: Mohorjeva. 
Rechtsinformationssystem des Bundes. (1955). „Staatsvertrag, betreffend die Wiederherstellung eines unabhängigen und demokratischen Österreich.“ Abgerufen am 2. April 2020. https:// www.ris.bka.gv.at/Dokumente/BgblPdf/1955_152_0/1955_152_0.pdf

Rechtsinformationssystem des Bundes. 2020a. „Bundes-Verfassungsgesetz." Abgerufen am 2. April 2020. https://www.ris.bka.gv.at/GeltendeFassung.wxe?Abfrage=Bundesnormen\&Gesetzesn ummer $=10000138$

Rechtsinformationssystem des Bundes. 2020b. „Volksgruppengesetz.“ Abgerufen am 2. April 2020. https://www.ris.bka.gv.at/GeltendeFassung.wxe?Abfrage=Bundesnormen\&Gesetzesnummer $=10000602$

Rechtsinformationssystem des Bundes. 2020c. „Staatsgrundgesetz über die allgemeinen Rechte der Staatsbürger." Abgerufen am 2.April 2020. https://www.ris.bka.gv.at/GeltendeFassung. wxe Abfrage $=$ Bundesnormen\&Gesetzesnummer $=10000006$.

spz.slo.at. 2013. „Intervju Florjana Lipuša v Dnevniku 27.12.2013.“ Abgerufen am 2. April 2020. https://spz.slo.at/sl/483/intervju-florjana-lipusa-v-dnevniku-27-12-2013

Uca, D. 2019. „"Grissgott” meets "Kung Fu”: Multilingualism, Humor, and Trauma in Saša Stanišić's Wie der Soldat das Grammofon repariert (2006).“ In Symposium 73 (3): 185-201. https://doi.org/10.1080/00397709.2019.1633806

Verein Aktion Mitarbeit. 2009. „mehr sprachig. Wegweiser zur Kommunikation im 21. Jahrhundert. Vortragsreihe Oktober 2008 - November 2009.“ Abgerufen am 2. April 2020. https: //www.okay-line.at/file/656/broschueremehrsprachig-.pdf

Volkan, V. 1999. Das Versagen der Diplomatie: Zur Psychoanalyse nationaler, ethnischer und religiöser Konflikte. Gießen: Psychosozial.

Volkan, V. 2005. Blindes Vertrauen. Großgruppen und ibre Führer in Krisenzeiten. Gießen: Psychosozial.

Winkler, J. 2020. „Volksbischof Josef/Joše.“ Kleine Zeitung, 3. Februar 2020.

Wutti, D. 2013. „Die Nähe zur Vergangenheit - Transgenerationale Übertragungen vor soziopolitischem Hintergrund.“ In Die Herstellung von Differenz. Zum Umgang mit Fremdheit in der transkulturellen Psychiatrie, Psychotherapie und Psychosomatik im deutschsprachigen Raum e.V. (DTPPP.) 6.-8. September 2012. Klinik Baselland Liestal, herausgegeben von T. Heise, T. Küchenhoff, I. Özkan und S. Golsabahi, 141-149. Berlin: VWB.

Wutti, D. 2014. „Der lange Hall der Vergangenheit - Zur psychotraumatischen „Transposition“ bei Kärntner Slowenen/innen.“ In Kärntner Jabrbuch für Politik 2014, herausgegeben von K. Anderwald, P. Filzmaier und K. Hren, 93-115. Klagenfurt/Celovec: Mohorjeva.

Wutti, D. 2017. „Erinnerung, Großgruppenidentität und politische Partizipation. Die Kärntner SlowenInnen im Spannungsfeld zwischen kultureller Unsicherheit und Sicherheit." In Lètopis 64, 2/2017: 238-251.

Wutti, D. 2020. „Dem Unaussprechbaren Sprache(n) geben? Mehrsprachigkeit und Trauma.“ In Mehrsprachigkeit. Interdisziplinäre Zugänge zu Mehrsprachigkeit und sozialer Teilhabe, herausgegeben von J. Meier und I. Dirim. Bad Heilbrunn: Julius Klinkhardt. In press. 